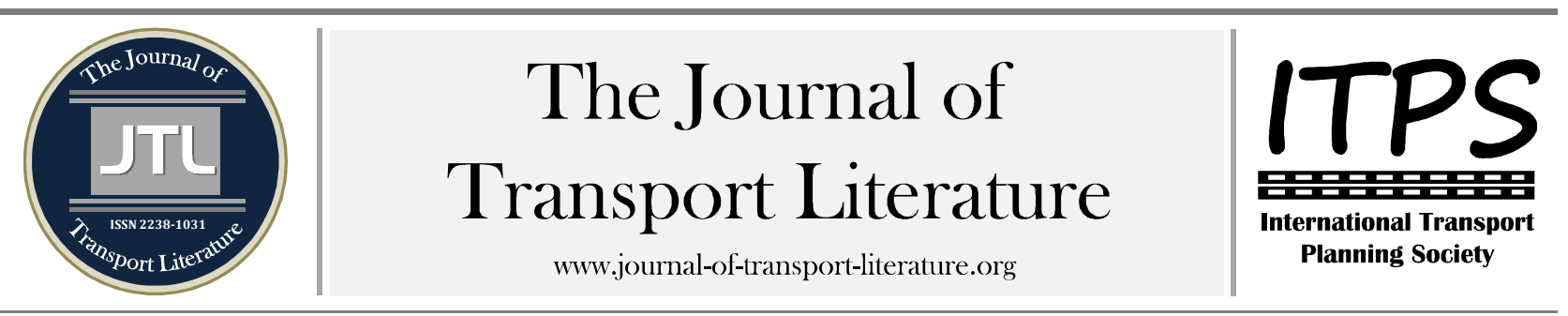

\title{
Solving the Integrated Schedule Generation and Fleet Assignment Problem: an ACo- Based Metaheuristic Approach
}

\author{
Daniel J. Caetano ${ }^{+}$; Nicolau D. F. Gualda \\ Universidade de São Paulo, São Paulo, Brasil
}

\section{Article Info}

Keywords:

air transportation

schedule generation

fleet assignment

metaheuristic

ant system

Submitted 12 Nov 2014; received in revised form 19 Jan 2015 accepted 20 Apr 2015

Licensed under

Creative Commons

CC-BY 3.0 BR.

\section{Abstract}

Traditionally, the initial steps on airline planning - Schedule Generation and Fleet Assignment problems are solved separately. This traditional approach usually leads to suboptimal solutions, since flight profitability - the decision criteria to schedule a flight - depends on what aircraft type will be used on that flight. On the other hand, the type of aircraft assigned to a flight will be different accordingly to the available scheduled flights. Because of this interdependence, airlines avoid complete redesign of their flight network, adopting a conservative approach and slightly improving existing suboptimal schedules over time. The integrated solution for both problems, albeit desirable, leads to large-scale models of the NP-Hard class. Some of the original linear constraints may become non-linear in the integrated problem, bringing further complexity to the solution process. This article presents a linear programming formulation of this integrated problem along with a heuristic approach, called MAGS, based on the ACO metaheuristic. Both the exact solution and the one provided by MAGS are obtained and compared for the case of a Brazilian airline. The results show the applicability of MAGS to real world cases, presenting solutions with objective function values distant no more than $6 \%$ of the optimum and much lower processing times than LP model on more complex configurations.

+ Corresponding author. Rua Prof. Celso Quirino dos Santos, 250 - Ap. 42-E - São Paulo / SP - Brasil - CEP 05353-030.

E-mail address: djcaetano@usp.br.

\section{Introduction}

The main objective of this paper is presenting a new heuristic model, based on the Ant Colony Optimization metaheuristic, designed to solve the integrated Schedule Generation Problem (SGP) and the Fleet Assignment Problem (FAP) for a regional airline. Additionally, this paper compares the heuristic performance and objective function values to an exact linear programming (LP) approach.

Along with the increasing passenger demand over the years, there was an increase in the frequency of flights. This fact, in addition to the increasing competition among airlines and operating limitations on main airports have direct influence on each flight aircraft type choice (Gramulha, 2011), requiring models to define new air transport networks accordingly to the evolution of demand and aiming at operating cost reduction (Hane et al. 1995).

Mathematical models that allow the definition of better air transport networks are relatively complex, pertaining to the NP-Hard class (Hane et al. 1995). Hence, there is a tendency to split the problem into stages (Rabetanety et al. 2006), simplifying the solution but possibly preventing the global optimal solution from being achieved, due to the inherent interaction among various steps inputs and outputs. Two of the several stages of the operational planning of an airline - SGP and FAP, can be solved using integrated linear programming models (Caetano and Gualda, 2012, 2011, 2010; Lohatepanont and Barnhart, 2006). Due to the NP-Hard characteristic of these models, their application to large scale problems is limited (Hane et al., 1995), leading to excessively large processing times on real life and large scale problems.

This paper presents both an exact LP model (Caetano and Gualda, 2011) and a new heuristic model that incorporates the Ant Colony Optimization metaheuristic to solve SGP and FAP as an integrated problem, avoiding model simplifications that could hinder its application to real world problems (Caetano and Gualda, 2012, 2011, 2010; Rabeanety et al. 2006). Such problems include the creation of entirely new schedules from scratch and operation limitations related to aircraft noise (Carvalho Júnior, Garavelli and Maroja, 2012) and arrival and departure restrictions (Alves and Fraga, 2012), also termed as "slots". The solutions obtained using the proposed heuristic are always close to the optima - $6 \%$ off in average - and the processing times are much lower on complex configurations - processing times at least 10 times faster than LP model. These characteristics allow the evaluation of multiple scenarios in shorter time.

Initially, a brief description of the traditional approach to solve FAP is presented, including a linear programming model to solve the integrated SGP/FAP. The following section presents the new Multiple Ant Colony Group System heuristic (MAGS), based on the Ant Colony Optimization (Dorigo and Stützle; 2004). Finally, a comparison between the results obtained through the metaheuristic and the optimal results obtained with the linear programming model is presented, followed by the conclusions of the study. 


\section{Airline Operational Planning}

The traditional approach to solving the fleet assignment models are based on a space-time network, where arrival or departure airports are represented by nodes. There are two basic types of arcs on this representation: flight leg arcs connecting nodes that represent different airports - or waiting time arcs - connecting nodes that represent different times at the same airport (Berge and Hoperstead, 1993 apud Sherali et al., 2006; Hane et al. 1995).

These classical models assume that the flight schedule is previously defined, with every flight being covered. Traditionally, they do not include operational restrictions at airports. To overcome these limitations, it is necessary to define a more comprehensive model. The model presented in this paper is based on the concept of space-time modelling (Berge and Hoperstead, 1993 apud Sherali et al. 2006; Hane et al. 1995), extended to cope with landing and departure slots by the addition of landing arcs that connect an arrival node to the first viable departure node.

The fleet assignment model can be integrated to schedule generation with the addition of a penalty for non served demand and relaxing the cover constraint so that not all flights must be assigned, as in the linear programming (LP) model presented below.

The objective function (expression 1) seeks to minimize the sum of lost revenues. The first term represents the difference between maximum revenue for the assigned aircraft and the revenue received from assigned passengers. The second term is associated to the lost revenue due to lost demand.

Linear Programming Model (Caetano and Gualda, 2012):

$$
\begin{aligned}
& {[\min ] \sum_{(i, j) \in L f}\left\{\left[\left(\sum_{f \in F} R_{i j} \cdot C^{f} \cdot x_{i j}^{f}\right)-R_{i j} \cdot p a_{i j}\right]+R_{i j} \cdot\left(d_{i j}-p a_{i j}\right)\right\}} \\
& \sum_{f \in F} x_{i j}^{f} \leq 1 \quad \forall(i, j) \in L f_{m}, \forall m \in M \\
& \sum_{o \mid(o, k) \in L} x_{o k}^{f}-\sum_{d \mid(k, d) \in L} x_{k d}^{f}=0 \quad \forall k \in N^{f}, \forall f \in F \\
& \sum_{(i, j) \in L t} x_{i j}^{f} \leq A^{f} \quad \forall f \in F \\
& \sum_{f \in F} \sum_{j \mid(i, j) \in L_{f}} x_{i j}^{f} \leq 1 \quad \forall i \in N r d \\
& \sum_{f \in F} \sum_{i \mid(i, j) \in L_{f}} x_{i j}^{f} \leq 1 \quad \forall j \in N r a \\
& \sum_{f \in F} C^{f} \cdot x_{i j}^{f}-p a_{i j} \geq 0 \quad \forall(i, j) \in L f \\
& d_{i j}-p a_{i j} \geq 0 \quad \forall(i, j) \in L f \\
& \sum_{(i, j) \in L f_{m}} d_{i j}-D_{m}=0 \quad \forall m \in M \\
& x_{i j}^{f} \in\{0,1\} \quad \forall(i, j) \in L f m \\
& x_{i j}^{f} \geq 0 \quad \forall(i, j) \in L \backslash L f m \\
& d_{i j} \geq 0 \quad \forall(i, j) \in L f \\
& p a_{i j} \geq 0 \quad \forall(i, j) \in L f
\end{aligned}
$$

where:

- $A^{f}$ is the number of aircraft of type f available;

- $C^{f}$ is the number of seats of aircraft of type $\mathrm{f}$;

- $d_{i j}$ is the number of potential passengers (demand) associated to the flight from node i to node j;

- $D_{m}$ is the unrestricted passenger demand on market m;

- $F$ is the set of all types of aircraft, indexed by f;

- $L$ is the set of arcs that represent the movement of aircraft, maintenance, waiting on the ground or wrap, indexed by $(i, j)$, being $i$ the source node and $j$ the destination node of the movement;

- $L f$ is the set of arcs that represent flight movements;

- $L f m$ is the set of arcs representing flights assigned to a market;

- $L t$ is the set of arcs whose origin time is equal to or less than $t$ and destination time is after $t$. The time $t$ is set to a valid time according to the problem;

- $M$ is the set of all markets, indexed by $\mathrm{m}$; each market defines a demand and a time window which limits which flights can serve this demand;

- $N^{f}$ is the set of all nodes for aircraft of type f, indexed by i, j, o, d or k, representing an airport at a specific time;

- Nra is the set of nodes with landing restrictions;

- $N r d$ is the set of nodes with departure restrictions;

- $p a_{i j}$ is the number of passengers associated to the flight from node i to node $\mathrm{j}$; 
- $R_{i j}$ is the unitary revenue for a passenger on the flight from node i to node j. Since $(\mathrm{i}, \mathrm{j})$ represent an specific flight including day and time - each flight may be associated with a specific unitary revenue;

- $x_{i j}^{f}$ is the number of aircraft of type f flowing through arc $(\mathrm{i}, \mathrm{j})$.

Expressions 2 to 4 represent the traditional cover, balance and number of aircraft restrictions (Berge and Hoperstead, 1993 apud Sherali et al. 2006; Hane et al. 1995).

Expressions 5 and 6 represent slot constraints, assuring that only one aircraft will depart or land on those nodes, respectively. Expressions 7 to 9 assure that each market demand will be associated to each flight and that the passengers of a flight will never be greater than the associated aircraft capacity.

The variables representing demanded flight arcs are binary, and are specified on expression 10 . All other arc variables are integers greater than or equal to zero, as stated on expression 11, 12 and 13.

\section{Ant Colony Modeling}

Flight scheduling and fleet assignment are traditionally solved using integer linear programming techniques such as node clustering and constraint relaxation. However, practical instances, representing the operation of major airlines, remain a challenge, given the computational complexity involved. On the other hand, there are many heuristics that are capable of finding very good solutions to several types of combinatorial problems (Abrahão, 2006), suggesting the search for heuristics that can provide appropriate solutions for the problem in lower processing times. The successful application to problems like Vehicle Routing Problem (VRP) and Aircraft Rotation Problem (ARP) draws attention to the metaheuristic known as Ant Colony Optimization (ACO), one of the many swarm intelligence metaheuristics (Teodorovic, 2008).

\subsection{ACO Applied to the Integrated SGP and FAP}

Although it was possible to adapt the basic ACO metaheuristic to solve the integrated flight schedule and fleet assignment problem, the results obtained through such approach were not satisfactory. Since the basic ACO leads to a single shortest path, it must be executed several times, assigning one aircraft at a time and removing the selected arcs from the list, leading to suboptimal solutions, with objective function values almost three times the optimal ones.

However, the problem has specific characteristics that can be taken into consideration to improve the ACO metaheuristic. Thus, an new ACO heuristic is proposed, called Multiple Ant Group System (MAGS). It incorporates elements of Multiple Ant Colony Optimization (MACO)(Vrancx and Nowé, 2006), Multiple Ant Colony System (MACS) and Elitist Ant System (EAS)(Dorigo and Stützle, 2004), as well as new elements not present on other ACO metaheuristic variants.

\subsection{Multiple Ant Group System - MAGS Heuristic}

MAGS is a multiple ant colony heuristic, such as MACS and MACO. As in MACO, a solution is represented by multiple ants; on the other hand, the number of ants that build a specific solution is previously known: there must be one ant per aircraft. The ants that compose a solution are called an ant group. A group may be composed of ants from different colonies and, similar to what is presented in MACS, each colony has a different objective function. This means that ants from each colony make decisions based on different criteria. In MACS, however, pheromones are identical for all colonies, which means it is substantially different from MAGS.

The proposed solution construction process is substantially different from the classical ACO, to reduce the number of invalid and unrealistic solutions. During the construction of a solution, the ants of a group will alternately choose graph arcs. The group's ant which will take the next step is randomly selected and whenever a flight arc is associated to an ant, this arc will be no longer available to other ants in the same group, ensuring the construction of solutions in which two or more aircraft do not share flights.

Additionally, when a flight is selected by an ant, part of the flight's market demand is also allocated, reducing the demand available to other flights that share the same market. This strategy avoids the association of ants to flight arcs for which the demand is no longer available in that solution. As the demand for each arc becomes dynamic during the construction of the solution, the problem presents similar characteristics to the dynamic routing in communication networks, as solved by the AntNet heuristic (Dorigo and Stützle, 2004). The exclusion of arcs and the demand allocation during the solution construction have relevant effects on the results, which are complementary to that provided by the repellent pheromones proposed on MACO, which continues to affect the selection probability of each arc.

Considering the described construction process, each ant group has the same role of a single ant in the basic ACO: the group of ants represents the complete objective function, each ant associated with a different term of it.

The MAGS algorithm starts with the creation of an initial "best" solution, represented by an ant group. Then the iteration, termed "season", starts. At each "season" several ant groups are generated and improved with local search methods, followed by the pheromones evaporation/update process. This iterative process is repeated for a number of "seasons" and then the algorithm ends.

As proposed by Dorigo and Stützle (2004), the nearest neighborhood solution can be adopted as an initial solution. On the addressed problem, the "nearest neighbor" was defined as the arc associated with the minimum revenue loss, avoiding waiting arcs whenever possible. The objective function value for this solution is used to determine the initial pheromone deposit on each arc.

Differently from the basic ACO, the initial pheromone deposit is not the same for all arcs. Arcs associated with smaller heuristic values must receive substantially more pheromones in the initial distribution than those associated to higher heuristic values. Considering the basic ACO probability equation (expression 14), an increased pheromone deposit value on arcs that have low heuristic value will also increase their likelihood of being chosen, at least in the initial heuristic stage.

$$
p_{i j}=\frac{\tau_{i j}^{\alpha} \cdot \eta_{i j}^{\beta}}{\sum_{l \mid(i, l) \in N_{i}} \tau_{i l}^{\alpha} \cdot \eta_{i l}^{\beta}}
$$

The probability equation adopted (expression 15), however, presents some additional parameters. The first one is $\phi_{i j}$, which represents the amount of pheromone of other colonies, as in MACO, with their respective coefficient $\gamma$. Additionally, 
the proposed parameter $\rho_{i j}$ reduces the probability of selecting a sequence of several unprofitable flights. The $\rho_{i j}$ value is always 1.0 for profitable flight, maintenance, and waiting arcs. For unprofitable flight arcs, its value starts as 1.0 but upon the addition of an unprofitable arc to the solution, the value of $\rho_{i j}$ is reduced by $50 \%$ for the next unprofitable flight arc. This value is only reset to 1.0 when a profitable flight is selected to compose the solution.

$$
p_{i j}=\frac{\tau_{i j}^{\alpha} \cdot \eta_{i j}^{\beta} \cdot \phi_{i j}^{-\gamma} \cdot \rho_{i j}}{\sum_{l \mid(i, l) \in N_{i}} \tau_{i l}^{\alpha} \cdot \eta_{i l}^{\beta} \cdot \phi_{i l}^{-\gamma} \cdot \rho_{i l}}
$$

The $\eta_{i j}$ value is proportional to the flight profit, given that there is demand available in the market. The $\eta_{i j}$ is made equal to $\tau_{i j}$ for repositioning flight arcs - which have no markets associated to them -, waiting arcs and maintenance arcs, since the heuristic value based on lost revenue on these arcs would be always non positive. The objective of this measure is also to reduce the myopic heuristic behavior, adding more emphasis on historical quality of the solutions containing a specific arc, which is represented by the pheromone deposit value.

After the initial pheromone is distributed, g ant groups are generated, but no changes are made on pheromones, as in the basic ACO. As the generation of the ant groups is completed, pheromone evaporation takes place, at a fixed rate, and then all $\mathrm{g}$ ant groups will update their pheromone trails. As in the EAS, the best solution will reinforce its own pheromone trail, leading to convergence toward that solution.

The pheromone deposit for each ant group is proportional to the objective function value, as in the basic ACO, but each ant of that group shall deposit only part of the group total pheromone: the amount of pheromone each ant of a group deposits is proportional to the ant's contribution to the quality of the solution represented by that group. The proposed distribution rule is defined by the expression 16, where $\tau_{f}$ is the deposit of each ant, with $\tau_{g}=1 / C g$, where Cg is the cost of the solution represented by the group, calculated through the objective function. $R_{f}$ is the revenue generated by that ant and $M R_{g}$ is the maximum revenue that could be generated by that group of ants.

$$
\tau_{f}=\tau_{g} \cdot\left\{0.5+\left[R_{f} / 2 . M R_{g}\right]\right\}
$$

This formulation guarantees that each ant will deposit a value not smaller than $50 \%$ of the deposit calculated for the group and also ensures that it will increase when the ant has a large contribution to the group total revenue.

It is important to notice that when each group finishes its solution construction, a local search is performed to improve that solution. This local search is divided into two steps: LS, which is quicker and handles all the solutions, and LS2, which is slower and processes only the solutions that have a value close to the optimum one or are too far from it. The LS is a procedure that removes sequences of two unprofitable flight arcs. This procedure also includes a corrective heuristic, which adjusts the solution so that each ant's terminal and initial airports are the same. The LS2 supplements LS, looking for profitable flights that could replace waiting arcs on each ant's path.

\subsection{Application and Results}

Both LP model and MAGS were applied to instances based on a domestic regional airline that carries 104 weekly flights and uses three ATR-42/300 aircraft (for 50 passengers each). Alternative flight networks were generated with different fleet configurations, involving Embraer 120 (for 30 passengers each), and Embraer 170 (for 70 passengers each). Some of the instances also include alternative flights for a new destination, expanding the base network to 164 weekly flights, plus new alternatives for repositioning flights.

The demand distribution adopted on each instance can be of three different types:

- Fixed: the demand associated to each flight is fixed at 50 passengers.

- Flight: the demand is associated to each flight and is the average demand per flight, based on values provided by the Brazilian Civil Aviation regulatory agency - ANAC (2007).

- Period: the demand between two airports associated to a period of day - morning or evening - is the average demand by day period, based on values provided by ANAC (2007).

The instances were solved by integer linear programming techniques through Gurobi Optimizer (C) software version 3, on an Intel Core2 Quad computer with 2GB of memory, using four processing cores and 200GB of available virtual memory. MAGS was implemented using Java SE version 6, running on the same equipment while using only one of the cores, since MAGS was not implemented using parallel programming.

Table 1 shows the results obtained by both the LP model and MAGS for some of the instances, given a weekly schedule. The values of the objective function represent the total lost revenue and, thus, the lower the value, the better the solution.

Analyzing the results, it is possible to notice that MAGS leads to results very close to those obtained by the exact model, with small standard deviations and much lower processing times - none of the instances took more than an hour on each run. The minimum values obtained, shown in Table 2, are even closer to the optimal ones: while the average values are distant by up to $6 \%$ of the optimum, the minima are no more than $3 \%$ greater than the optimum value for each case. Pratical differences between LP and MAGS solutions are usually relative to one or two flights. The small standard deviation shows that the heuristic usually converges to solutions of similar objective function values, if not to the same solution.

MAGS average processing time per arc was of 0.04 seconds. The processing times, though not directly linear to the number of arcs, are very low compared to processing times of the LP model solved with the Gurobi Optimizer (C), even without a parallel implementation for MAGS. 
Table 1: Exact model and MAGS average results.

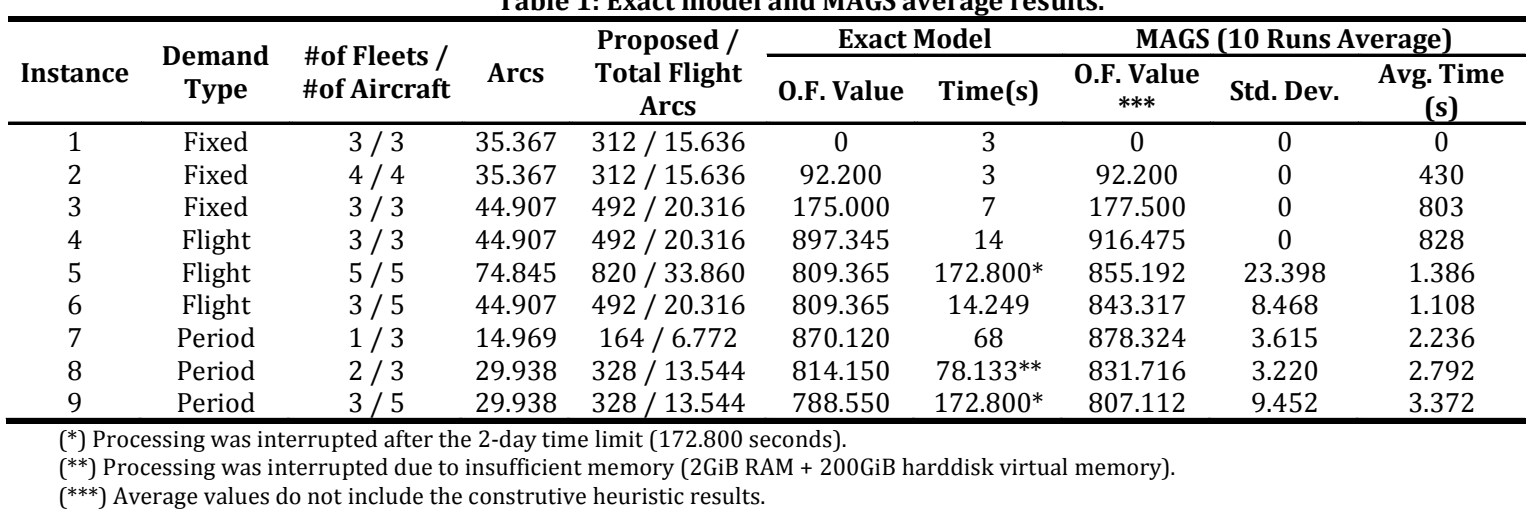

MAGS can be fine-tuned to improve these results when considering specific characteristics of each configuration - type of demand, for instance -, but the aim of this analysis was the evaluation of the performance and quality of generated solutions with a generic set of heuristic parameters. Unlike some other integrated heuristic approaches, this is not an interactive multi-step heuristic - both SGP and FAP are solved at the same time. Also, the heuristic results show that it provides good solutions in low processing times for instances with different input characteristics.

\section{Conclusion}

This study presented and compared two models to solve the flight schedule and the fleet assignment problems in an integrated way. Both models incorporate the same objective function and constraints, including real world operational restrictions such as slots at airports, allowing the evaluation and definition of new flight networks.

One of the models relies on Linear Programming, which is capable to solve relatively small instances, due to the NP-Hard characteristic of the integrated problem. The other, a new heuristic approach called MAGS - Multiple Ant Group System - is based on the ACO metaheurisic and addressed to solve large scale problems in low processing times. MAGS presents a distinctive way to determine the initial pheromone level on each arc, as well as alternative rules for the construction of solutions, each one being represented by a group of multiple ants. In addition, the multiple ant solution representation required a new rule for pheromone updating, which is also shown.

Both models were applied to instances related to a Brazilian airline. The exact model reached optimal solutions only for relatively small instances. MAGS reached very close results to the optimal ones in lower processing times, addressing the possibility to utilize it to solve real world problems.

Due to the strategic/tactical nature of the presented models, none of them take into account complex aircraft maintenance or flight connections to attend a specific market; these extensions are suggested for future works. There is room for improvement on MAGS heuristic also, adding more characteristics of the problem as heuristic criteria, in order to reduce even more the gap between the obtained results and the optimal ones.

\section{References}

Abrahão, F.T.M., \& Gualda, N.D.F. (2006) Meta-Heurística Colônia de Formigas para Solução do Problema de Programação de Manutenção Preventiva de Frotas de Veículos. Proceedings of the XIV Panamerican Conference of Traffic and Transportation Engineering, Las Palmas de Gran Canaria, Spain.

Alves, C. J. P., \& Fraga, R. (2012) Capacidade do lado aéreo dos aeroportos brasileiros. Journal of Transport Literature, 6(4), $178-189$.

ANAC (2007). Anuário Estatístico da Agência Nacional de Aviação Civil vol. I.

Caetano, D.J., \& Gualda, N.D.F. (2010). A Flight Schedule and Fleet Assignment Model. Proceedings of 12th World Conference on Transport Research, Lisboa, Portugal.

Caetano, D.J., \& Gualda, N.D.F. (2011). Um Modelo Integrado para a Programação de Voos e Alocação de Frotas. Transportes, 19 , $16-24$.

Caetano, D. J., \& Gualda, N.D.F. (2012). An Integrated Model for Flight Scheduling and Fleet Assignment Based on the Ant Colony MetaHeuristic. Proceedings of XVII PANAM - Pan-American Conference of Traffic and Transportation Engineering and Logistics, Santiago, Chile.

Carvalho Júnior, E. B., Garavelli, S. L., \& Maroja, A. M. (2012). Análise dos efeitos do ruído aeronáutico em zonas residenciais circunvizinhas ao Aeroporto Internacional de Brasília. Journal of Transport Literature, 6(4), 59-81.

Dorigo, M., \& Stützle, T. (2004). The Ant Colony Optimization. Massachussets: Bradford Book.

Gramulha, R.(2011). Escolha do tamanho da aeronave pelas empresas aéreas. Journal of Transport Literature, 5(4), 232-239.

Hane, C.; Barnhart, C.; Johnson, E.; Marsten, R.; Nemhauser, G.; Sigismondi, G. (1995). A fleet assignment problem: Solving a large-scale integer program, Technical report, Georgia Institute of Technology. Report Series 92(4).

Rabetanety, A., Calmet, J., \& Schoen, C. (2006). Airline Schedule Planning Integrated Flight Schedule Design and Product Line Design (Master's thesis). Universität Karlsruhe, Karlsruhe, Germany.

Sherali, H.D., Bish, E.K., \& Zhu, X. (2006). Airline fleet assignment concepts, models, and algorithms. European Journal of Operational Research , 172, 1-30.

Teodorovic, D. (2008). Swarm Intelligence Systems for Transport Engineering: Principles and Applications, Transportation Research Part C: Emerging Technologies, 16(6), 651-667.

Vrancx, P., \& Nowé, A. (2006). Using Pheromone Repulsion to Find Disjoint Paths. In Dorigo, M., Gambardella, L.M., Birattari, M., Martinoli, A., Poli, R.; \& Stützle, T. (Eds.) Ant Colony Optimization and Swarm Intelligence 5th International Workshop. (pp. 522-523) Brussels (BE): ANTS. 